\title{
Potencial da cinza do bagaço da cana-de-açúcar como material de substituição parcial de cimento Portland
}

\author{
Marcos O. de Paula ${ }^{1}$, Ilda de F. F. Tinôco ${ }^{2}$, Conrado de S. Rodrigues ${ }^{3}$, Elizabeth N. da Silva ${ }^{4}$ \& Cecília de F. Souza $^{2}$
}

\begin{abstract}
RESUMO
Este trabalho, voltado para a avaliação do potencial da cinza do bagaço da cana-de-açúcar (CBC) como material de substituição parcial do cimento Portland em argamassa, objetivou apresentar opção viável para a destinação deste resíduo, cuja quantidade gerada aumentará significativamente nos próximos anos, em decorrência da ampliação do setor de produção de álcool combustível; além disso, o emprego da CBC como adição mineral, substituindo parte do cimento em argamassas e concretos, contribui para a redução do impacto ambiental desses materiais, em boa parte decorrente da produção do cimento. O procedimento experimental abordou não só caracterização da CBC mas também a avaliação, através de ensaios físicos e mecânicos, em que os resultados mostraram que o bagaço apresenta rendimento de CBC de $10 \%$, com a cinza sendo composta de $84 \%$ de $\mathrm{SiO}_{2}$ e $5 \%$ de Carbono. A sílica na CBC apresenta-se na fase amorfa e nas fases cristalinas de cristobalita e quartzo. Os índices de atividade pozolânica comprovam a reatividade da CBC. Do ponto de vista da resistência à compressão, argamassas com teores de CBC entre 0 e 30\% indicaram a possibilidade de substituição de até $20 \%$ do cimento pela CBC.
\end{abstract}

Palavras-chave: manejo de resíduos, materiais de construção, desenvolvimento sustentável

\section{Potential of sugarcane bagasse ash as a partial replacement material for Portland cement}

\begin{abstract}
This study is focused on the evaluation of the effects of the partial replacement of Portland cement by sugarcane bagasse ash $(\mathrm{CBC})$ in mortars. The main objective was to find a suitable destination for an agricultural residue generated in an increasing amount in Brazil, as a result of the boom of the use of ethanol as an alternative fuel to gasoline. Also, the use of $\mathrm{CBC}$ as a mineral admixture in mortars and concretes contributes to a decrease in the environmental impact of these materials related to cement production. Experimental techniques were applied both for the $\mathrm{CBC}$ characterization and for the evaluation of its use as a mineral admixture in mortars, based on mechanical and physical tests. The yield of CBC from sugarcane bagasse burning was $10 \%$ (weight basis). The $\mathrm{CBC}$ presented a $\mathrm{SiO}_{2}$ content of $84 \%$ and a carbon content of $5 \%$. Silica presented both amorphous and crystalline (cristobalite and quartz) structure. The pozzolanicity index applied indicates the reactivity of the $\mathrm{CBC}$. Concerning the compression strength, the results from tests with mortars with up to $30 \%$ of $\mathrm{CBC}$ content indicated the viability of the partial substitution of cement by up to $20 \%$ of the CBC considered.
\end{abstract}

Key words: solid waste management, building materials, sustainable development

\footnotetext{
1 Engo. Civil, Estudante de Doutorado, DEA/UFV, Viçosa, MG, Fone: (031) 9172-5297, E-mail: modep@vicosa.ufv.br.

2 Enga . Agrícola, Prof. Associada, DEA/UFV, Viçosa, MG. Fone: (31) 3899-1884, E-mail(s): iftinoco@ufv.br; cfsouza@ufv.br.

3 Engo. Civil, Prof. do DEC/CEFET, MG. Fone: (31) 3319-6826, E-mail: crodrigues@civil.cefetmg.br

${ }^{4}$ Enga. Florestal, Estudante de Doutorado, DEF/UFV. Viçosa, MG. Fone: (31) 3891-5295, E-mail: e neire@yahoo.com.br
} 


\section{INTRODUÇÃO}

A necessidade de geração de energia a partir de fontes renováveis vem impulsionando a produção de álcool etanol a partir da cana-de-açúcar. O Brasil se posiciona, atualmente, como o maior produtor mundial de açúcar e álcool e maior exportador mundial de açúcar. O Proálcool, Programa Nacional do Álcool, é o maior programa comercial de utilização de biomassa para a produção de energia no mundo e representou a iniciativa de maior sucesso mundial, na substituição de derivados de petróleo no setor automotivo, mediante o uso do álcool como combustível único nos veículos movidos a álcool hidratado; ainda hoje há cerca de 4 milhões de veículos que utilizam exclusivamente este derivado da cana como combustível, representando $40 \%$ da frota nacional. E não se deve esquecer o importante papel desempenhado na solução do problema da octanagem da gasolina, substituindo o chumbo tetraetila, altamente prejudicial à saúde humana, na mistura gasolina-álcool (gasohol), hoje aceita e usada em praticamente todo o mundo.

Durante a extração do caldo da cana-de-açúcar é gerada grande quantidade de bagaço (aproximadamente 30\% da cana moída), biomassa de suma importância como fonte energética. Cerca de $95 \%$ de todo o bagaço produzido no Brasil são queimados em caldeiras para geração de vapor gerando, como resíduo, a cinza de bagaço, cuja disposição não obedece, na maior parte dos casos, a práticas propícias, podendo-se configurar em sério problema ambiental. Constituída, basicamente, de sílica, $\mathrm{SiO}_{2}$, a cinza do bagaço de cana-de-açúcar (CBC) tem potencial para ser utilizada como adição mineral, substituindo parte do cimento em argamassas e concretos (Cordeiro et al., 2008).

A utilização pela construção civil de resíduos gerados em outros setores da economia é vantajosa não apenas em virtude do aumento da atividade industrial e, conseqüentemente, de subprodutos mas, sobretudo, devido à redução da disponibilidade de matérias-primas não renováveis, tão necessárias às atividades da construção civil convencional. Grande parte dos resíduos gerados pode ser reciclada, reutilizada, transformada e incorporada, de modo a produzir novos materiais de construção e atender à crescente demanda por tecnologia alternativa de construção mais eficiente, econômica e sustentável (Savastano, 2003).

Dentre os resíduos se destacam as cinzas minerais oriundas de diferentes atividades agroindustriais, que apresentam altas porcentagens de sílica e de outros óxidos, podendo ser então utilizadas como pozolanas. A propriedade da pozolana é a sua capacidade de reagir com o hidróxido de cálcio liberado durante o processo de hidratação do cimento, formando compostos estáveis de poder aglomerante, tais como os silicatos e aluminatos de cálcio hidratados (Oliveira et al., 2004).

As pesquisas realizadas sobre o assunto estão concentradas na cinza da casca de arroz, que apresenta teores de $\mathrm{SiO}_{2}$ usualmente superiores a 90\% (John et al., 2003); contudo, investigações demonstram que as cinzas de bagaço de canade-açúcar podem ter o mesmo poder de utilização requerendo, entretanto, estudos mais aprofundados.
Ante o exposto, esta pesquisa teve como objetivo avaliar o potencial de utilização da cinza do bagaço da cana-de-açúcar como aditivo mineral na produção de pastas e argamassas de cimento Portland.

\section{MATERIAL E MÉTODOS}

A CBC foi obtida do bagaço da cana-de-açúcar (BC), originária da Usina Jatiboca (Urucânia, MG). O BC foi coletado e queimado em mufla, durante 6 horas, a $600{ }^{\circ} \mathrm{C}$; após a queima, pôde-se observar uma camada superficial de cinza de cor clara sobre o restante do material, que se caracterizava por cinza de coloração preta e composição heterogênea, compreendendo restos de bagaço não queimados e partículas de carvão; desta forma, tornou-se conveniente uma nova queima para homogeneização da amostra e redução do teor de carbono (responsável pela coloração escura); a segunda queima da CBC foi realizada por 3 horas, a $700{ }^{\circ} \mathrm{C}$, seguida de resfriamento natural.

A composição química e a estrutura cristalina da CBC foram determinadas, respectivamente, com os ensaios de espectroscopia de fluorescência de raios-X (equipamento EDX-700 da marca Shimadzu) e difração de raios-X (difratômetro de pó SEIFERT-FPM GmbH operando com radiação de CuKa $(\alpha=1,5418 \AA)$ a $40 \mathrm{kV}$ e $40 \mathrm{~mA})$. O teor de carbono foi definido através da perda de massa a $700{ }^{\circ} \mathrm{C}$.

Para estudo das características granulométricas da CBC, esta foi submetida a diferentes períodos de moagem em um moinho de bolas, com as distribuições granulométricas estabelecidas por difração a laser (método de Fraunhöffer) e as áreas de superfície específicas por adsorção de $\mathrm{N}_{2}$ (método de $\mathrm{S}_{\mathrm{BET}}$ ) empregando-se o aparelho ThermoAnalytical QsurfM3. Para a realização desses ensaios se coletaram amostras após moagem, por 0, 30, 60, 120, 180, 300, 420, 540 e $660 \mathrm{~min}$.

$\mathrm{Na}$ produção das pastas e argamassas foram empregadas as seguintes taxas de substituição parcial do cimento por CBC: 0, 10, 20 e 30\%. As relações entre cimento - CBC nas argamassas e a nomenclatura adotada para as pastas e argamassas, são: C1 (100-0), C2 (90-10), C3 (80-20) e C4 (70-30), em que os valores representam os percentuais de cimento e CBC; foram usados o cimento Portland CPV ARI PLUS da marca Barroso e o traço 1:3, com areia normal brasileira.

Para se avaliar a influência da adição de CBC na argamassa, realizaram-se ensaios de tempo de pega inicial e final, resistência a compressão, índice de atividade pozolânica, determinação da massa específica, absorção de água por imersão e índice de vazios, ambos os ensaios foram realizados com base na Associação Brasileira de Normas Técnicas. O experimento foi desenvolvido adotando-se delineamento experimental inteiramente casualizado, constituído de 4 tratamentos, ou seja: 3 níveis de adição de CBC e um testemunho (100\% cimento), com 3 repetições. Os melhores níveis de adição de CBC foram avaliados com base no teste de Tukey para todas as combinações, aos 7 e 28 dias. 


\section{RESULTADOS E DISCUSSÃO}

O bagaço usado apresenta um rendimento de cinza de $10 \%$, em massa. A composição química é mostrada na Tabela 1.

Tabela 1. Composição química da CBC realizada por espectroscopia de fluorescência de raios-X

\begin{tabular}{cc}
\hline Composto químico & Composição (\%) \\
$\mathrm{SiO}_{2}$ & 83,707 \\
$\mathrm{Fe}_{2} \mathrm{O}_{3}$ & 6,537 \\
$\mathrm{~K}_{2} \mathrm{O}$ & 6,146 \\
$\mathrm{CaO}$ & 1,183 \\
$\mathrm{TiO}_{2}$ & 1,162 \\
$\mathrm{SO}_{3}$ & 0,682 \\
$\mathrm{ZrO}_{2}$ & 0,303 \\
$\mathrm{Cr}_{2} \mathrm{O}_{3}$ & 0,094 \\
$\mathrm{MnO}$ & 0,081 \\
$\mathrm{Sc}_{2} \mathrm{O}_{3}$ & 0,040 \\
$\mathrm{ZnO} 0$ & 0,037 \\
$\mathrm{~V}_{2} \mathrm{O}_{5}$ & 0,029 \\
\hline
\end{tabular}

Neste caso, a cinza apresentou teor de sílica de aproximadamente 84 e 5\% de carbono, valores semelhantes foram encontrados por Villar-Cociña et al. (2006). O material, antes da queima e após a queima, é mostrado na Figura 1. A fase ativa dos diferentes aditivos minerais é composta basicamente de sílica, ou seja, quanto maior o teor de sílica ativa melhor será a atividade pozolânica da cinza (Massazza, 1998).

Segundo Cook (1986), a presença de cerca de 20\% de carbono na cinza, não afeta significativamente a resistência a compressão, porém o decréscimo na resistência passa a ser percebido quando são empregadas cinzas com teores de carbono elevados, decréscimo este devido à queda na quantidade de sílica ativa correspondente.
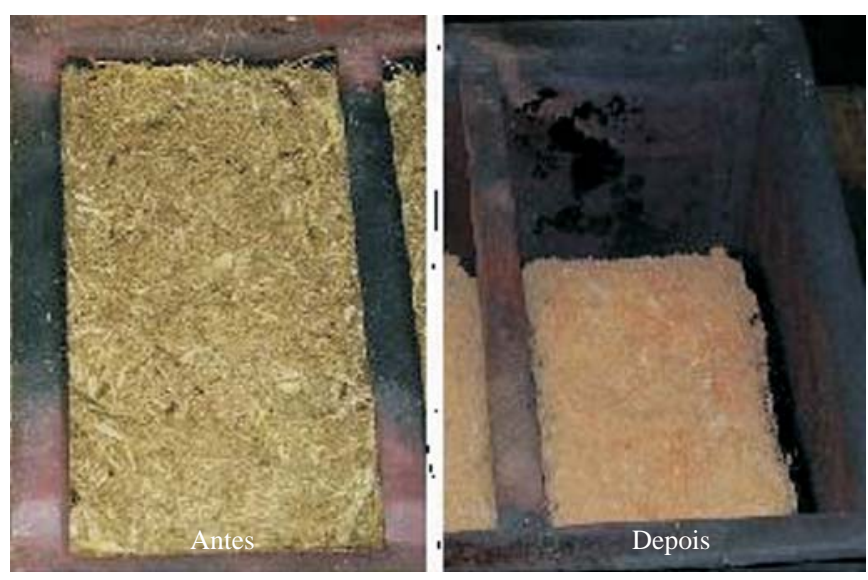

Figura 1. Bagaço da cana-de-açúcar antes e após a queima a $700{ }^{\circ} \mathrm{C}$

As fases cristalinas da CBC são descritas pelo espectro de difração de raios-X mostrado na Figura 2, na qual segundo Villar-Cociña et al. (2003), o halo entre $2 \theta=06$ e $18^{\circ}$ caracteriza a fase amorfa. Os picos mostram a presença de fases cristalinas da sílica nas formas de: cristobalita (C) $\left(2 \theta=36,5^{\circ}\right)$ e quartzo $(\mathrm{Q})\left(2 \theta=21^{\circ}, 2 \theta=27^{\circ}\right.$ e $\left.2 \theta=39^{\circ}\right) ;$ além desses, foram detectados picos relacionados à estrutura da muscovita $(\mathrm{M})\left(2 \theta=24,5^{\circ}\right)$ o que se deve, provavelmente, à contaminação do bagaço por partículas do solo, assim como o quartzo. A presença desses contaminantes pode ser evitada com a lavagem do bagaço ou com a disposição em local apropriado.

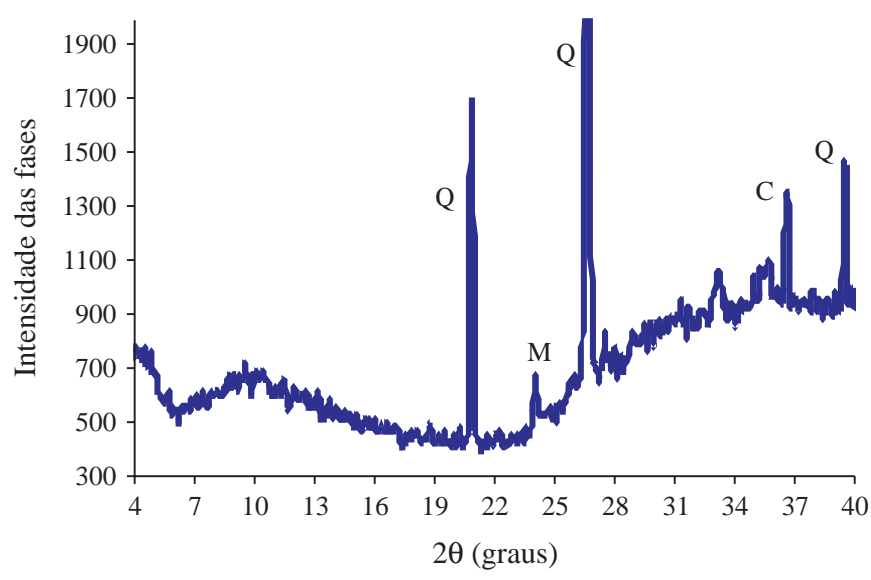

Figura 2. Análise de difração de Raio-X da CBC

Os resultados encontrados para a composição granulométrica e área de superfície demonstraram que a cinza em estudo é composta de partículas com tamanho entre 1 e $14 \mathrm{~mm}$ e com uma área de superfície da ordem de $24 \mathrm{~m}^{2} \mathrm{~g}^{-1}$.

No que diz respeito ao ensaio de tempo de pega inicial e final, as adições de 10, 20 e 30\% de CBC não causaram adiantamento nem retardo do início da pega, o que era de se esperar, uma vez que a reação pozolânica ocorre em estágio mais adiantado da hidratação do cimento (7 e 15 dias após a mistura); assim, o tempo de início de pega não detectaria influência da atividade pozolânica. As adições de 10, 20 e $30 \%$ de CBC acarretaram retardo de 10 min no intervalo de tempo entre os tempos de pega; este retardo se deve à redução do teor de cimento nas combinações.

Os resultados encontrados para os índices de atividade pozolânica (IAP) (Tabela 2) comprovam a reatividade da CBC, uma vez que todas as combinações apresentaram valores para o IAP superiores ao valor mínimo de 75\% estabelecido pela norma técnicas. Do exposto, a CBC pode ser classificada como pozolana.

Notou-se que o acréscimo de CBC nos corpos-de-prova acarretou um gradativo escurecimento na coloração dos

Tabela 2. Resistência média à compressão em MPa da argamassa aos 28 dias e respectivos índices de atividade pozolânica (IAP) para as diferentes misturas de cimento e CBC

\begin{tabular}{ccc}
\hline Mistura & $\begin{array}{c}\text { Resistência Média* } \\
\text { (MPa) aos 28 dias }\end{array}$ & IAP (\%) \\
C1 & 47,8 & 100 \\
C2 & 48,0 & 100 \\
C3 & 46,9 & 99 \\
C4 & 40,7 & 86 \\
\hline
\end{tabular}

* Média de 3 corpos de prova 
mesmos. As Figuras 3A e 3B ilustram os resultados de resistência a compressão realizados nos corpos-de-prova, em função da interação entre o teor de substituição de cimento por cinza de CBC, aos 7 e 28 dias de idade.

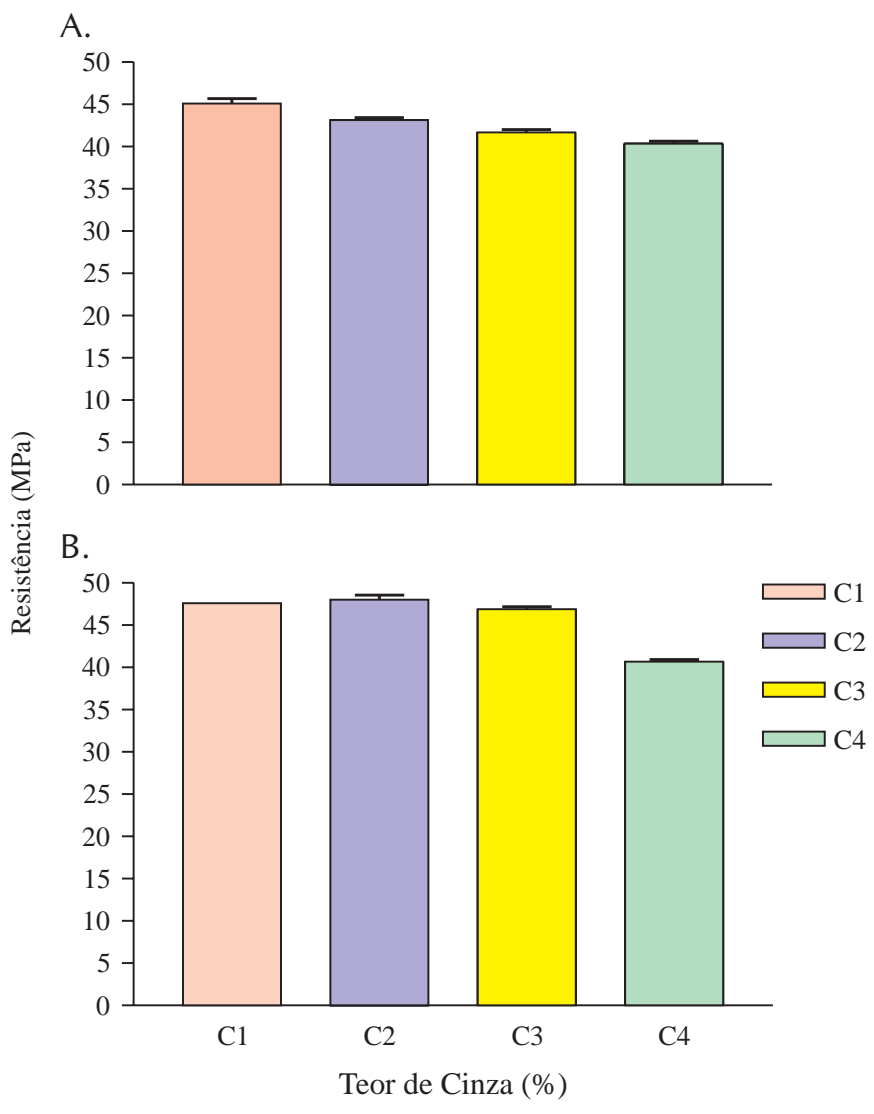

Figura 3. (A.) Resistência a compressão aos 7 dias nas argamassas incorporadas com resíduos; (B.) idem aos 28 dias

A análise de variância aplicada aos dados de resistência a compressão mostrou, a nível de $5 \%$ de probabilidade pelo teste Tukey, alto grau de significância para combinações aos 7 e 28 dias. Aplicado aos dados de resistência a compressão aos 7 e 28 dias na argamassa de cimento com adição de CBC, o teste de Tukey mostrou que aos 7 dias de idade, independentemente da adição de CBC considerada, o tratamento C1 apresentou-se estatisticamente diferente em relação aos demais. As combinações C2 e C3 não foram diferentes entre si, assim como as combinações C3 e C4, conforme apresentado na Tabela 3. Observa-se que a resistência a compressão das argamassas aos 7 dias é inversamente proporcional ao teor de cinza adicionado.

Tabela 3. Valores médios da resistência a compressão (fcm) aos 7 e 28 dias de idade na argamassa sem $\mathrm{CBC}$ e com adição de $\mathrm{CBC}$ no níveis de 10,20 e $30 \%$ (C2, C3 e C4 respectivamente)

\begin{tabular}{ccccc}
\hline \multirow{2}{*}{ Idade } & \multicolumn{5}{c}{ Mistura } \\
\cline { 2 - 5 } $\mathrm{fc} 7$ & C1 & C2 & C3 & C4 \\
$\mathrm{fc} 28$ & $45,10 \mathrm{a}$ & $43,13 \mathrm{~b}$ & $41,7 \mathrm{~b} \mathrm{c}$ & $40,37 \mathrm{c}$ \\
\hline
\end{tabular}

* As médias seguidas de pelo menos uma mesma letra não diferem entre si, a nível de $5 \%$ de probabilidade pelo teste Tukey
Considerada aos 28 dias, a análise estatística mostrou que os maiores valores de resistência foram alcançados pelas combinações C1, C2 e C3, estatisticamente iguais entre si (Tabela 3). Este emparelhamento da resistência aos 28 dias indica que a reação pozolânica tem início entre 7 e 15 dias após a mistura, quando a hidratação do cimento se apresenta já em estado avançado, conforme observado por Rodrigues (2006); tais resultados apontam a possibilidade de se substituir até $20 \%$ do cimento Portland por cinzas de bagaço de cana-de-açúcar, sem prejuízo da resistência a compressão.

Nas Figuras 4A, B e C se apresentam os resultados dos ensaios de absorção de água por imersão, índice de vazios e massa específica dos corpos-de-prova de argamassa, em função do teor de substituição aos 28 dias de idade.

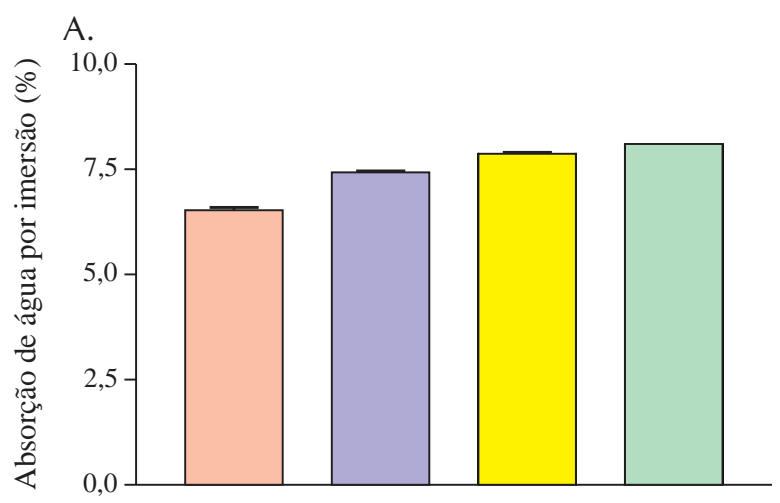

B.
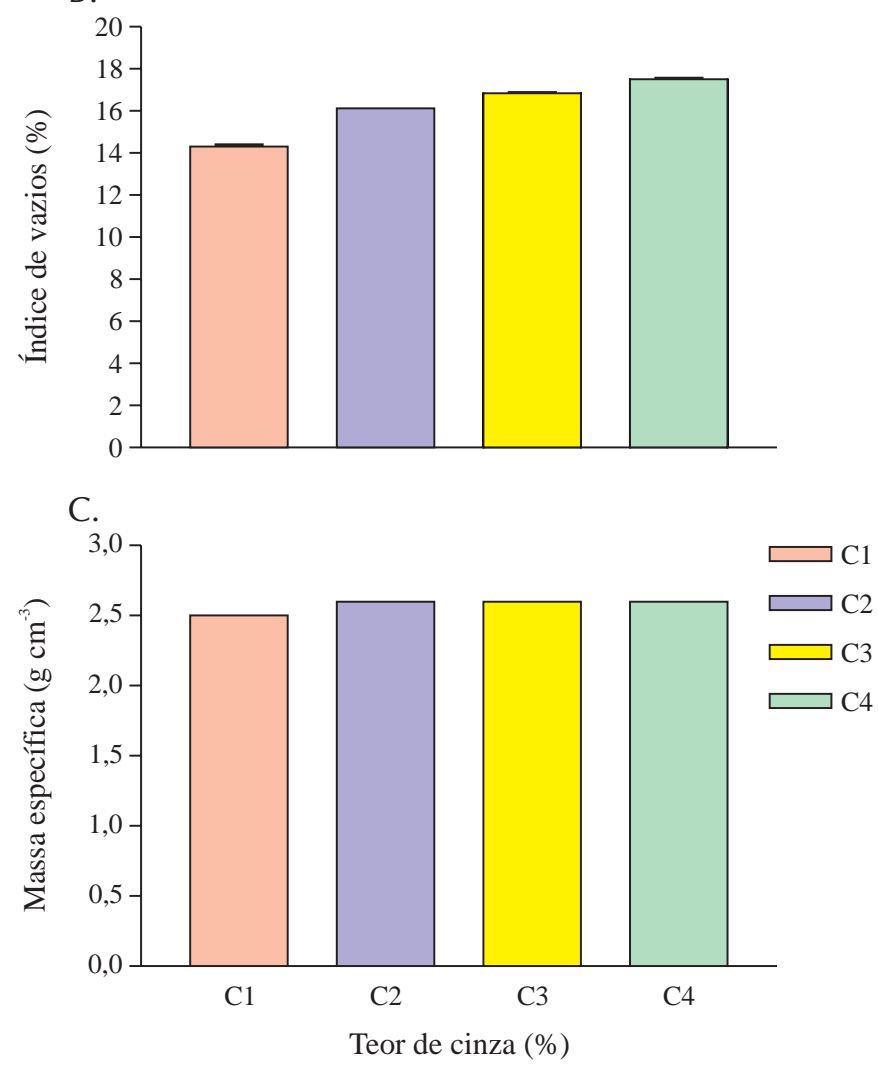

Figura 4. (A) Absorção de água por imersão, (B) índice de vazios e (C) massa específica, aos 28 dias, nas argamassas confeccionadas com diferentes concentrações de cimento e cinza de bagaço de cana-de-açúcar 
Observa-se, tanto para a absorção de água por imersão quanto para o índice de vazios, que as combinações resultaram em uma função crescente, donde se conclui que as argamassas com maiores teores de cinza tendem a ser mais porosas, o que justifica os maiores valores de absorção. Segundo Barbosa et al. (1998), este fato pode ser resolvido controlando-se o fator água/cimento de cada combinação estudada. Com relação à massa específica, observou-se um pequeno aumento com a adição de CBC (0,5\% em todas as combinações).

Com base nas análises de variância, pode-se observar influência significativa do teor de adição de CBC nos resultados de absorção de água por imersão e índice de vazios. Aplicado aos dados de absorção de água por imersão e do índice de vazios aos 28 dias na argamassa de cimento com adição de CBC, o teste de Tukey mostrou que não houve interação significativa entre as combinações estudadas (Tabela 4).

Tabela 4. Valores médios da absorção de água e índice de vazios aos 28 dias de idade na argamassa sem e com adição de CBC

\begin{tabular}{lcrrrr}
\hline \multirow{2}{*}{ Parâmetros } & & \multicolumn{4}{c}{ Combinações } \\
\cline { 3 - 6 } & C1 & C2 & C3 & C4 \\
Absorção de água (\%) & Média & 6,6 a & 7,5 b & 7,8 c & 8,0 d \\
Índice de Vazios (\%) & Média & 14,4 a & 16,3 b & 16,8 c & 17,3 d \\
\hline
\end{tabular}

* As médias nas linhas, seguidas de pelo menos uma mesma letra, não diferem entre si, a nível de $5 \%$ de probabilidade, pelo teste Tukey

A análise de variância mostrou que não houve influência significativa do teor de adição de CBC nos resultados de massa específica. O que se pode depreender dessa análise, é que a substituição parcial do cimento Portland por até 30\% de cinzas na mistura não provocou qualquer modificação significativa nos valores da massa específica.

\section{CONCLUSÕES}

1. O bagaço de cana-de-açúcar utilizado apresentou rendimento de cinza de bagaço de cana-de-açúcar (CBC) de $10 \%$, com teor $\mathrm{SiO}_{2}$ de $84 \%$.

2. Esses teores, combinados com previsões de aumento na geração deste resíduo em decorrência da expansão do setor sucroaçucareiro no Brasil, apresentam a CBC como fonte viável de adição mineral de cimentos, dependendo das características da sílica presente.

3. No caso, a sílica na CBC apresentou-se tanto na fase amorfa quanto nas fases cristalinas de cristobalita e quartzo.

4. Argamassas com maiores teores de cinza foram mais porosas e com maior absorção de água.

5. Os índices de atividade pozolânica comprovaram a reatividade da CBC.

6. Os resultados dos ensaios de compressão aos 28 dias indicaram a viabilidade de substituição de até $20 \%$ de cimento por CBC sem prejuízo da resistência.

7. A obtenção de cinzas com maiores teores de sílica reativa, principalmente por meio de procedimentos de queima melhor ajustados, pode permitir maiores teores de substitui- ção ou melhorias mais significativas nas propriedades físicas e mecânicas das argamassas.

\section{AGRADECIMENTOS}

À Universidade Federal de Viçosa, nos seus diversos seguimentos, em especial ao Departamento de Engenharia Agrícola e Ambiental.

À FAPEMIG, pelo financiamento do projeto e pela bolsa de Iniciação cientifica e de apoio técnico, e a CAPES, pela concessão da bolsa de estudos.

\section{LITERATURA CITADA}

Barbosa, M. F., Lima, E., Pires Sobrinho, C. W. A. Estudo de argamassas com adições de cinza de casca de arroz e cinza de cana-de-açúcar. Encontro Nacional de Tecnologia do Ambiente Construído Qualidade no Processo Construtivo, 7, 1998, Florianópolis. Anais... Florianópolis: UFSC/ANTAC, 5p. 1998.

Cook, D. J. Rice husk ash. In: Swamy, R. N. (ed). Concrete techonology and design - v.3: Cement replacement materials. London: Blackie \& Son Ltd, 1986. 170p.

Cordeiro, G. C.; Toledo Filho, R. D.; Fairbairn, E. M. R.; Tavares, L. M. M. Pozzolanic activity and filler effect of sugar cane bagasse ash in Portland cement and lime mortars. Cement \& Concrete Composites, v.30, p.410-418, 2008.

John, V. M.; Cincotto, M. A.; Silva, M. G. Cinza e aglomerantes alternativos. In: Freire, W. J.; Beraldo, A. L. Tecnologia e materiais alternativos de construção. Campinas: UNICAMP, 2003. cap.6, p.145-190.

Massazza, F. Pozzolana and pozzolanic cements. In: Hewlett, P. C. (ed.), Lea's chemistry of cement and concrete, 4th edition, London: Arnold Publishers, 1998. p.485-500.

Oliveira, M. P.; Nobrega, A. F.; Campo, M. S.; Barbosa, N. P. Estudo do caulim calcinado como material de substituição parcial do cimento Portland. Conferencia Brasileira de Materiais e Tecnologias Não-Convencionais: Habitação e infraestrutura de interesse social Brasil - NOCMAT 2004, Pirassununga. Anais... Pirassununga: USP, 2004. 15p.

Rodrigues, C. S.; Ghavami, Khosrow; Stroeven, P. Porosity and water permeability of rice husk ash-blended cement composites reinforced with bamboo pulp. Journal of Materials Science, v.41, p.6925-6937, 2006.

Savastano, Jr., Warden, P. G. Special theme issue: Natural fibre reinforced cement composites. Cement \& Concrete Composites, v.25, n.5, p.517-624, 2003.

Villar-Cociña, E.; Valencia-Morales, E.; Gonzales-Rodrigues, R.; Hernandez Ruíz, J. Kinetics of the pozzolanic reaction between lime and sugar cane straw ash by electrical conductivity measurement: A kinetic-diffusive model. Cement and Concrete Research, v.33, p. 517-524, 2003.

Villar-Cociña, E.; Valencia-Morales, E.; Sanches Rojas M. I. An Evalution of different kinetics models for determining the kinetic coefficients in sugar cane-straw - ash/lime system. Advances in Cement Research, v.18, p. 17-26, 2006. 